\title{
Nonlinear System Identification of Folding Fins with Freeplay Using Direct Parameter Estimation
}

\author{
Zhi-Sai Ma $\mathbb{D}^{1,2}$ Bo Wang, ${ }^{3}$ Xin Zhang, ${ }^{4}$ and Qian Ding $\mathbb{D}^{1,2}$ \\ ${ }^{1}$ School of Mechanical Engineering, Tianjin University, Tianjin 300350, China \\ ${ }^{2}$ Tianjin Key Laboratory of Nonlinear Dynamics and Control, Tianjin 300350, China \\ ${ }^{3}$ Beijing Institute of Structure and Environment Engineering, Beijing 100076, China \\ ${ }^{4}$ Beijing Institute of Electronic System Engineering, Beijing 100854, China
}

Correspondence should be addressed to Qian Ding; qding@tju.edu.cn

Received 4 September 2019; Revised 8 October 2019; Accepted 26 October 2019; Published 15 November 2019

Academic Editor: Teng Wu

Copyright (c) 2019 Zhi-Sai Ma et al. This is an open access article distributed under the Creative Commons Attribution License, which permits unrestricted use, distribution, and reproduction in any medium, provided the original work is properly cited.

Folding fins are widely adopted in missiles for the efficient use of space during storage and transportation, while nonlinear behavior of freeplay is inevitable due to the factors such as mismachining tolerance, assembly error, and abrasion. The problem of nonlinear system identification of folding fins with freeplay is considered in this paper. A direct parameter estimation method which can identify the nonlinear system with freeplay under base excitation is proposed and subsequently applied to establish the nonlinear dynamic model of a folding fin. The best set of coefficients is selected by using the significance test, allowing the proposed method to detect and locate the most relevant nonlinearities of the practical structure. Experimental results demonstrate that the proposed method is able to decouple the linear and nonlinear dynamics of a nonlinear structure and estimate natural frequencies of the derived linear system along with nonlinear internal forces in one computational step, even if no a priori knowledge of the type of nonlinearities is given.

\section{Introduction}

Folding fins are widely adopted in missiles for the efficient use of space during storage and transportation. These fins are packed in folded state before launching and unfolded immediately after launching. Although most folding fins have a complex hinge consisting of dowels, torsional springs, and stoppers, nonlinear behavior of freeplay is inevitable due to the factors such as mismachining tolerance, assembly error, and abrasion $[1,2]$. Because of the existence of freeplay nonlinearities, both static and dynamic characteristics of folding fins are changed, especially the vibration and aeroelasticity characteristics [3-6]. For example, structural nonlinearities make the results of ground vibration test distorted and nonlinear phenomena (such as limited cycle oscillations, chaotic motions, and bifurcations) occur in some flight conditions.

To address the demand for flight vehicles with everincreasing technological and environmental performances, researchers try more and more regularly to investigate the nonlinear dynamic analysis of folding fins/wings [1-8]. On the one hand, two- or three-degree-of-freedom airfoils with freeplay nonlinearity were generally used to reveal nonlinear phenomena and validate corresponding nonlinear analysis methods $[3-6,9,10]$. These studies can offer useful insights into what is happening to the folding structures with freeplay, but they cannot be directly used to solve practical engineering problems, as real folding structures are flexible components with more degrees of freedom. On the other hand, substructure synthesis methods were used to couple the constructed hinge model and the other parts of the folding fins, and to establish an order-reduced model of the entire structure $[1,2,11-13]$. However, in most cases the types and values of nonlinear characteristics of the hinges are unknown and difficult to obtain.

Due to the complexity of the problem, it is usually difficult to build the explicit model of a folding fin by exclusively using mechanism analysis, and there is also no guarantee that the model will accurately represent its nonlinear dynamic characteristics. Therefore, nonlinear system identification is 
considered in this work to establish the nonlinear dynamic model of a folding fin. Nonlinear system identification is a vast research field and different methods including linearization, time- and frequency-domain methods, timefrequency analysis, modal methods, black-box modeling, and numerical model updating have been developed in the past $[14,15]$. Time-domain methods exclusively rely on processing time series and allow users to model the system in an intuitive way. Typical time-domain approaches comprise restoring force surface (RFS) analysis [16, 17], nonlinear autoregressive moving average with exogenous input (NARMAX) modeling [17-19], and nonlinear subspace identification methods $[20,21]$. Obviously, there is a concentrated nonlinearity in the hinge, and the folding fin is a continuous structure with localized structural nonlinearities. Therefore, direct parameter estimation (DPE) methods [22], as a class of extended RFS methods, are further developed to identify the structural nonlinearities of folding fins in this work.

The remainder of the paper is organized as follows: Section 2 introduces the experimental setup of a folding fin. Section 3 proposes a direct parameter estimation method which can identify the nonlinear system with freeplay under base excitation. Section 4 presents the identification results and the corresponding interpretation. Section 5 summarizes the study.

\section{Experimental Setup}

2.1. Experimental Folding Fin. The experimental folding fin with adjustable freeplay, as shown in Figure 1, consists of an outboard wing, a folding hinge, an inner wing, and a rudder shaft. The rudder shaft and the inner wing are manufactured together, and freeplay exists only between the inner wing and the outboard wing [1]. Six accelerometers are used to measure the acceleration responses of the structure at six distributed positions, and they are sequentially numbered from no. 1 to no. 6, as shown in Figure 1. During the experiment, the bottom of the rudder shaft is fixed on a rigid support structure by bolts, and the rigid support structure is fixed on a shaking table, as shown in Figure 2. The base excitation is measured by an accelerometer (no. 0) attached to the rigid support structure.

2.2. Nonlinear Detection. The frequency response function (FRF) summarizes most of the information necessary to specify the dynamics of a structure, and FRF distortions can be used to provide indications of the structural nonlinearity. In this section, the base sine-sweep test is adopted to ascertain if nonlinearity exists in the structural behavior. Based on the excitation signal and the corresponding response signal acquired by the no. 1 accelerometer, Figure 3 shows the frequency response characteristics of the folding fin without freeplay. The curves of the upward and downward sweeps are similar to each other and no jump phenomenon is observed, which indicates that the structure is linear. Regarding the folding fin with freeplay, significant differences and jumps can be observed by comparing its upward and downward sweep curves, as shown in Figure 4 . The jump occurs on the right-hand side of the first

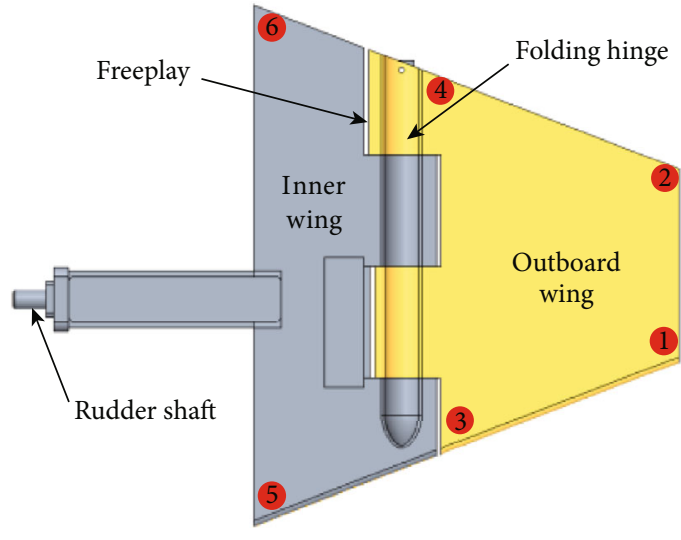

Figure 1: Sketch of the experimental folding fin.

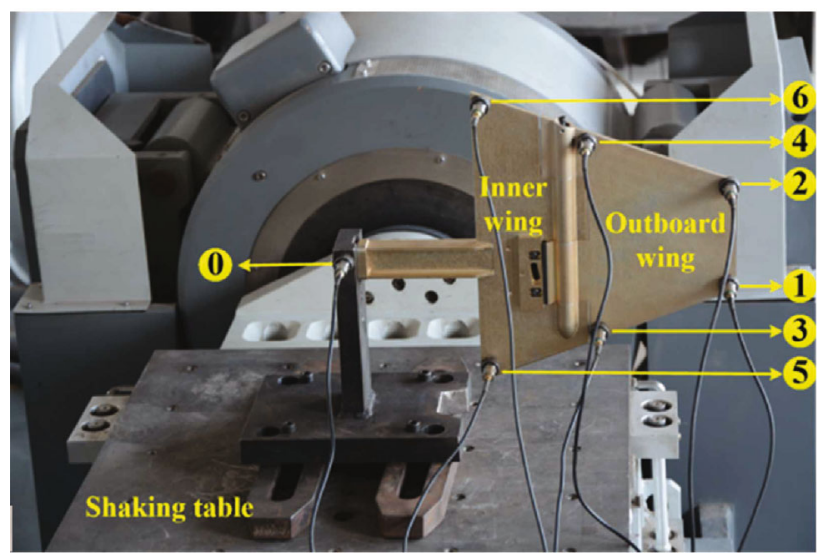

FIgURe 2: Photo of the experimental set-up.

resonance peak, and the system can be referred to as hardening. Similarly, sine-sweep tests of the folding fin with various forms of freeplay have been carried out. Results demonstrate that the resonance peaks move to lower frequencies with increasing freeplay and the freeplay has much larger influence on the first resonance peak (bending mode) than the second one (torsional mode).

\section{Direct Parameter Estimation}

3.1. Identification Theory. For a general mechanical structure, it can be discretized into lumped masses connected by restoring force links to each other and to the ground [22], as illustrated in Figure 5. The mass is assumed to be concentrated at $N$ measurement points, with $m_{i}$ designating the mass at point $i$. Each point $i$ is assumed to be connected to each other point $j$ by a link $l_{i j}$, and to the ground by a link $l_{i i}$.

The internal forces in the links depend on the relative displacements and velocities of the masses at each end of the links. Therefore, the force in link $l_{i j}$ is $f_{i j}\left(\delta_{i j}, \dot{\delta}_{i j}\right)$, with $\delta_{i j}=$ $x_{i}-x_{j}$ designating the relative displacement of mass $m_{i}$ to mass $m_{j}$, and $\dot{\delta}_{i j}=\dot{x}_{i}-\dot{x}_{j}$ the corresponding relative velocity. The force in the link to ground $l_{i i}$ is $f_{i i}\left(\delta_{i i}, \dot{\delta}_{i i}\right)$, where $\delta_{i i}=x_{i}$ and $\dot{\delta}_{i i}=\dot{x}_{i}$. 


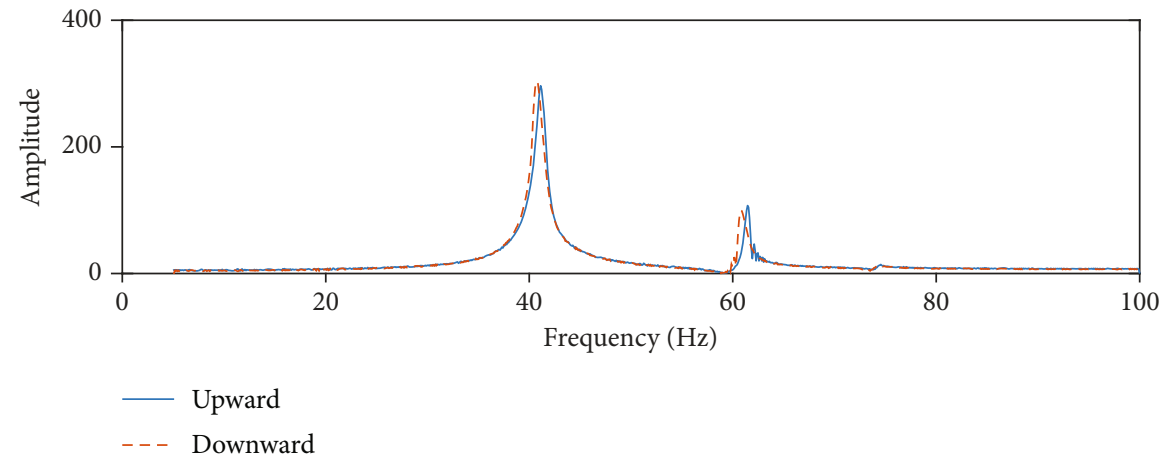

FIGURE 3: Frequency response characteristics of the folding fin without freeplay.

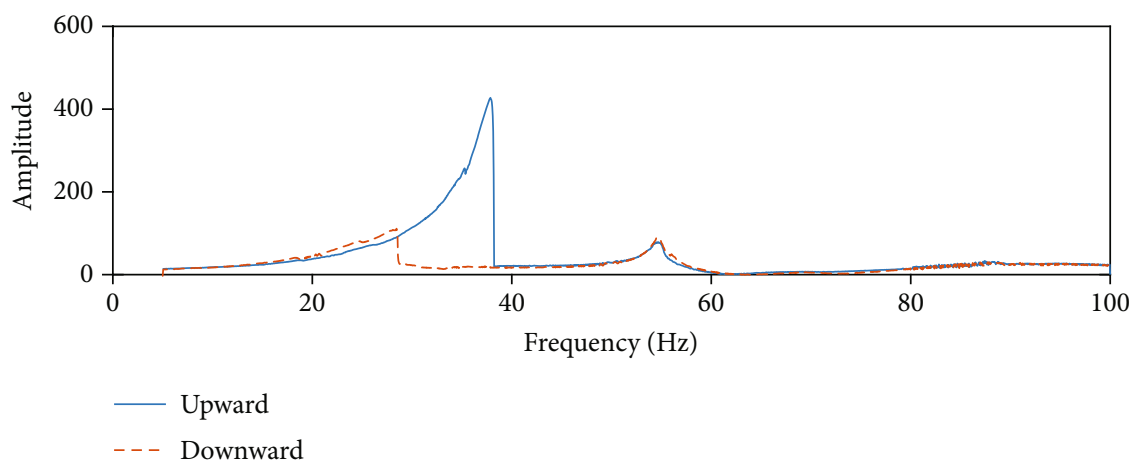

FIGURE 4: Frequency response characteristics of the folding fin with freeplay.

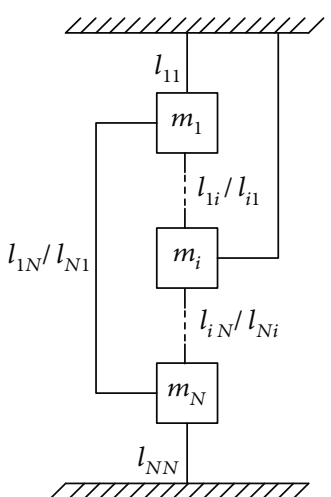

FIGURE 5: Link model of the $N$-degree-of-freedom system.

If an external force $y_{i}$ is applied to each mass $m_{i}$, the equations of motion of the $N$-degree-of-freedom system can be obtained as follows:

$$
m_{i} \ddot{x}_{i}+\sum_{j=1}^{N} f_{i j}\left(\delta_{i j}, \dot{\delta}_{i j}\right)=y_{i}, \quad i=1, \cdots, N
$$

Furthermore, if the responses of the system are induced by motions of its base support, then we have

$$
m_{i} \ddot{\delta}_{i i}+\sum_{j=1}^{N} f_{i j}\left(\delta_{i j}, \dot{\delta}_{i j}\right)=-m_{i} \ddot{x}_{0}, \quad i=1, \cdots, N
$$

with $\ddot{x}_{0}$ designating the acceleration of the base support and $\ddot{\delta}_{i i}=\ddot{x}_{i}-\ddot{x}_{0}$.

It has been proven that freeplay nonlinearity can be approximated by a polynomial expansion [10]; in this work, polynomial representation is used for internal forces $f_{i j}$ and (2) can be rewritten into the following form:

$$
m_{i} \ddot{\delta}_{i i}+\sum_{j=1}^{N} \sum_{k=0}^{p} \sum_{l=0}^{q} a_{(i j) k l}\left(\delta_{i j}\right)^{k}\left(\dot{\delta}_{i j}\right)^{l}=-m_{i} \ddot{x}_{0}, \quad i=1, \cdots, N
$$

As the lumped masses concentrated at different measurement points are difficult to obtain, (3) can be further rewritten as

$$
\sum_{j=1}^{N} \sum_{k=0}^{p} \sum_{l=0}^{q} \tilde{a}_{(i j) k l}\left(\delta_{i j}\right)^{k}\left(\dot{\delta}_{i j}\right)^{l}=-\ddot{x}_{i}, \quad i=1, \cdots, N
$$

with $\tilde{a}_{(i j) k l}=\left(1 / m_{i}\right) a_{(i j) k l}$. Obviously, the coefficients $\tilde{a}_{(i j) k l}$ can be directly estimated by least squares estimation.

As links $l_{i j}$ and $l_{j i}$ are the same, the symmetry relation $f_{i j}\left(\delta_{i j}, \dot{\delta}_{i j}\right)=-f_{j i}\left(\delta_{j i}, \dot{\delta}_{j i}\right)$ holds, then we have $m_{i} \tilde{a}_{(i j) k l}\left(\delta_{i j}\right)^{k}$ $\left(\dot{\delta}_{i j}\right)^{l}=-m_{j} \tilde{a}_{(j i) k l}\left(\delta_{j i}\right)^{k}\left(\dot{\delta}_{j i}\right)^{l}$. Therefore, the lumped mass $m_{j}$ can be obtained by 


$$
m_{j}=(-1)^{k+l+1} \frac{m_{i} \tilde{a}_{(i j) k l}}{\tilde{a}_{(j i) k l}} .
$$

Without loss of generality, by assuming that the mass at the first measurement point is $m_{1}=1$, the mass at other points can be estimated by using (5). In this way, a priori estimate of the mass is no longer required and an equivalent identified system of the true structure can be established. Substituting the estimates of the coefficients $\tilde{a}_{(i j) k l}$ and the corresponding masses $m_{i}$ into (3), we have the matrix motion equation of the $N$-degree-of-freedom system in the following general form:

$$
M \ddot{\Delta}+C \dot{\Delta}+K \Delta+F(\Delta, \dot{\Delta})=-M \ddot{X}_{0}
$$

where $\Delta=\left[\delta_{11}, \delta_{22}, \cdots, \delta_{N N}\right], \ddot{X}_{0}=\left[\ddot{x}_{0}, \ddot{x}_{0}, \cdots, \ddot{x}_{0}\right]$, the mass matrix is $M=\operatorname{diag}\left\{1, m_{2}, \cdots, m_{N}\right\}$, the damping matrix $C$, the stiffness matrix $K$, and the nonlinear internal force matrix $F(\Delta, \dot{\Delta})$ can be assembled based on (3).

The identified model of (6) can represent a system with a finite number of excited modes. The first $N$ natural frequencies $\left(p_{1}, \cdots, p_{N}\right)$ of the derived linear system can be computed by solving the following eigenvalue problem:

$$
\left|K-p^{2} M\right|=0 \text {. }
$$

3.2. Model Validation. In order to avoid estimating the overdetermined coefficients, a significance test is used to select the best set of coefficients, as follows:

Significance $\left\{a_{(i j) k l}\right\}=\frac{\sigma^{2}\left\{a_{(i j) k l}\left(\delta_{i j}\right)^{k}\left(\dot{\delta}_{i j}\right)^{l}\right\}}{\sigma^{2}\left\{\sum_{j=1}^{N} \sum_{k=0}^{p} \sum_{l=0}^{q} a_{(i j) k l}\left(\delta_{i j}\right)^{k}\left(\dot{\delta}_{i j}\right)^{l}\right\}}$,

with $\sigma^{2}\{\cdot\}$ designating the variance of the term in brace. Generally, the term $a_{(i j) k l}\left(\delta_{i j}\right)^{k}\left(\dot{\delta}_{i j}\right)^{l}$ can be removed from the identified model, if Significance $\left\{a_{(i j) k l}\right\}$ is smaller than a specified tolerance. Otherwise, this term should be consid- ered during the data-based modeling. The inclusion of different terms allows the identified model to detect and locate the most relevant nonlinearities [17]; for example, if the term $a_{(i j) 30}\left(\delta_{i j}\right)^{3}\left(\dot{\delta}_{i j}\right)^{0}$ appears, one may infer the presence of a cubic stiffness nonlinearity between measurement points $i$ and $j$.

In order to quantify the achievable identification accuracy, a normalized mean square error (MSE) [22] is used to measure the goodness of fit between the estimated acceleration $\left(\widehat{\ddot{x}}_{i}\right)$ and its experimental counterpart $\left(\ddot{x}_{i}\right)$

$$
\mathrm{MSE}_{i}=\frac{\left\|\ddot{x}_{i}-\widehat{\ddot{x}}_{i}\right\|^{2}}{L \sigma^{2}\left\{\ddot{x}_{i}\right\}}, \quad i=1, \cdots, N
$$

where $L$ is the length of samples.

\section{Identification Results and Interpretation}

4.1. Measurement and Acquisition. The six accelerometers, as shown in Figure 1, are used to measure the acceleration responses of the folding fin with freeplay, and its first two modes are considered in this work. The base excitation is white noise band-limited into a $10-80 \mathrm{~Hz}$ interval to excite only the first two modes, as shown in Figure 6. The acceleration signals, sampled at $2048 \mathrm{~Hz}$, are $4 \mathrm{~s}$ long, producing 8192 sample-long versions of the vibration measurements. The velocity and displacement signals are obtained by integrating the acceleration measurements using the trapezium rule and band-pass filtered into a $20-200 \mathrm{~Hz}$ interval to eliminate spurious components from the integration and retain a sufficient number of harmonics in the data. Figure 7 shows the filtered vibration signals of the folding fin at the first measurement point.

4.2. Two-Measurement-Point Identification Results. The proposed direct parameter estimation method is used to identify the folding fin based on the excitation signal given in Figure 6 and the corresponding response signals at the first two measurement points. The best set of coefficients are selected by using the significance test with the predefined tolerance of 0.001 and the identified model is obtained as

$$
\begin{aligned}
& \left(\begin{array}{cc}
1 & 0 \\
0 & -0.2191
\end{array}\right)\left(\begin{array}{l}
\ddot{\delta}_{11} \\
\ddot{\delta}_{22}
\end{array}\right)+\left(\begin{array}{cc}
21.9153 & -17.8562 \\
2.7006 & 0.6924
\end{array}\right)\left(\begin{array}{l}
\dot{\delta}_{11} \\
\dot{\delta}_{22}
\end{array}\right)+10^{4}\left(\begin{array}{cc}
3.3006 & 0.3726 \\
1.7110 & -2.4871
\end{array}\right)\left(\begin{array}{l}
\delta_{11} \\
\delta_{22}
\end{array}\right) \\
& +\left(\begin{array}{c}
159.5266 \dot{\delta}_{11}^{3}-1.8400 \times 10^{4} \dot{\delta}_{11}^{2} \delta_{11}-1.8881 \times 10^{7} \dot{\delta}_{11} \delta_{11}^{2}+1.7992 \times 10^{9} \delta_{11}^{3}-1.1311 \times 10^{4} \dot{\delta}_{12}^{3}+1.9993 \times 10^{11} \delta_{12}^{3} \\
-35.0139 \dot{\delta}_{22}^{3}+3.0000 \times 10^{3} \dot{\delta}_{22}^{2} \delta_{22}+3.8512 \times 10^{6} \dot{\delta}_{22} \delta_{22}^{2}-2.4542 \times 10^{8} \delta_{22}^{3}+1.1311 \times 10^{4} \dot{\delta}_{21}^{3}-1.9993 \times 10^{11} \delta_{21}^{3}
\end{array}\right) \\
& =-\left(\begin{array}{cc}
1 & 0 \\
0 & -0.2191
\end{array}\right)\left(\begin{array}{c}
\ddot{x}_{0} \\
\ddot{x}_{0}
\end{array}\right) \text {. }
\end{aligned}
$$




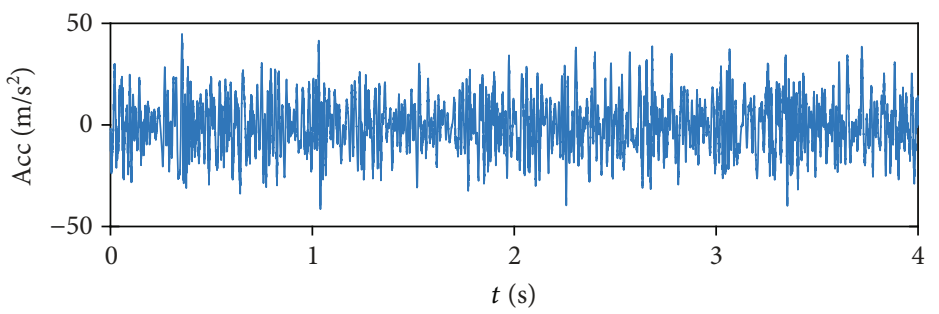

Figure 6: Base excitation signal of the folding fin.
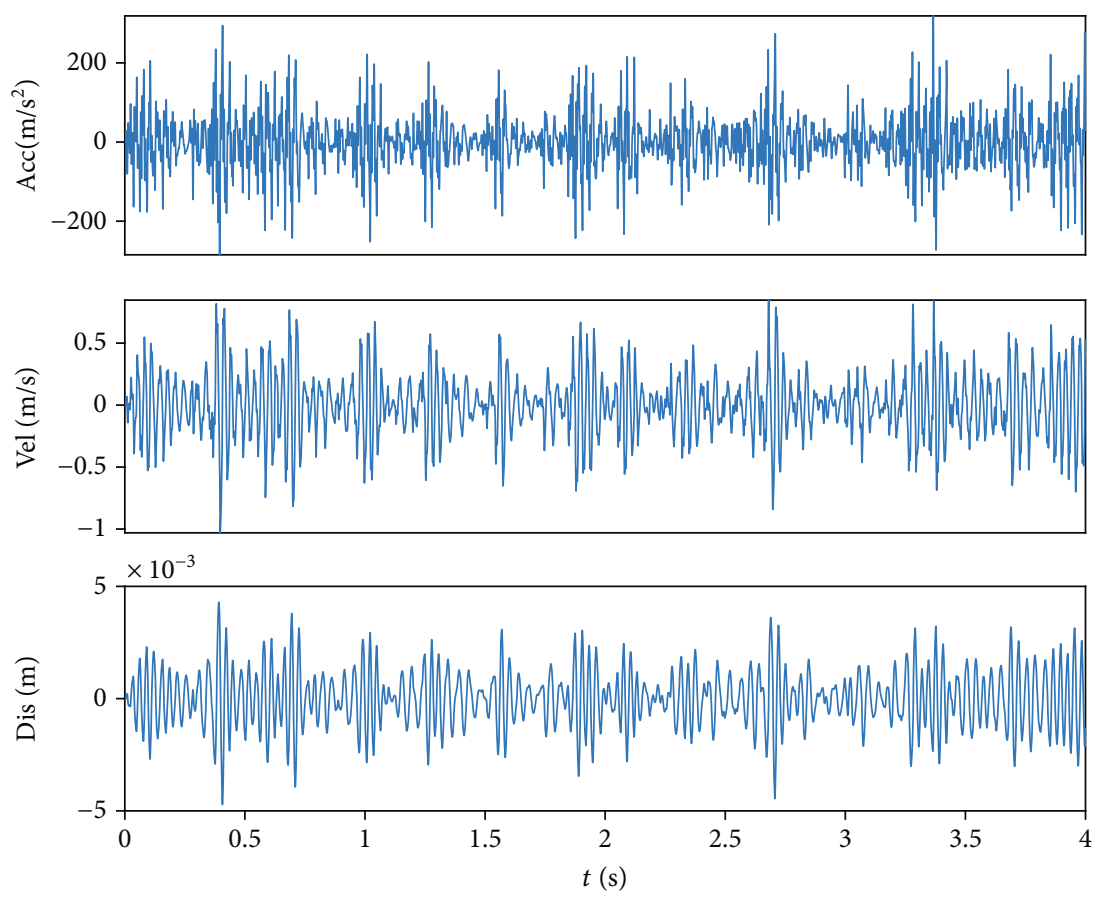

FIGURE 7: Filtered vibration signals of the folding fin at the first measurement point.

Furthermore, the mass and stiffness matrices of (10) are extracted and the first two natural frequencies of the derived linear system can be computed through (7) as $p_{1}=30.531 \mathrm{~Hz}$ and $p_{2}=52.718 \mathrm{~Hz}$. Figure 8 depicts the experimental acceleration signals at the first two measurement points and their estimated counterparts. The normalized mean square errors between experimental acceleration signals and their estimated counterparts are $\mathrm{MSE}_{1}=0.3038$ and $\mathrm{MSE}_{2}=0.3784$.

Similarly, the first two natural frequency estimates of the derived linear system and the corresponding MSEs are obtained based on the data from different combinations of two measurement points, as shown in Table 1. Evidently, the identification results vary slightly with the choice of data at different measurement points. Nevertheless, it may lead to unreliable identification results based on the data at two measurement points located on the inner wing, such as no. 5 and no. 6 . The reason is that freeplay exists only between the inner wing and the outboard wing, and the base excitation signal is acquired at the bottom of the rudder shaft. The relative relationships between the measurement point at the inner wing and the excitation point at the rudder shaft do not directly reflect the freeplay nonlinearity, as the rudder shaft and the inner wing are manufactured together. Therefore, the information of the freeplay nonlinearity cannot be well captured by exclusively using measurements from the inner wing. In other words, some accelerometers should be placed at the outboard wing in order to better capture the freeplay nonlinearity, in case base excitation is applied to the folding fin.

4.3. Comparative Results and Discussions. The identification results can be further improved by using the data at more measurement points. In this section, three-, four-, five-, and six-measurement-point-based identification is sequentially carried out, and the best set of coefficients is selected by using the significance test with the predefined tolerance of 0.001 . Table 2 depicts the MSEs of a part of the identification results by selecting the data at two, three, four, five, and six measurement points. As shown in Table 2, the fitting errors decrease gradually with the increase of the number of measurement points. However, as the number of measurement points increases, the dimensionality of the identified model increases. In other words, more and more poles will be computed through (7). In order to indicate the number of present 


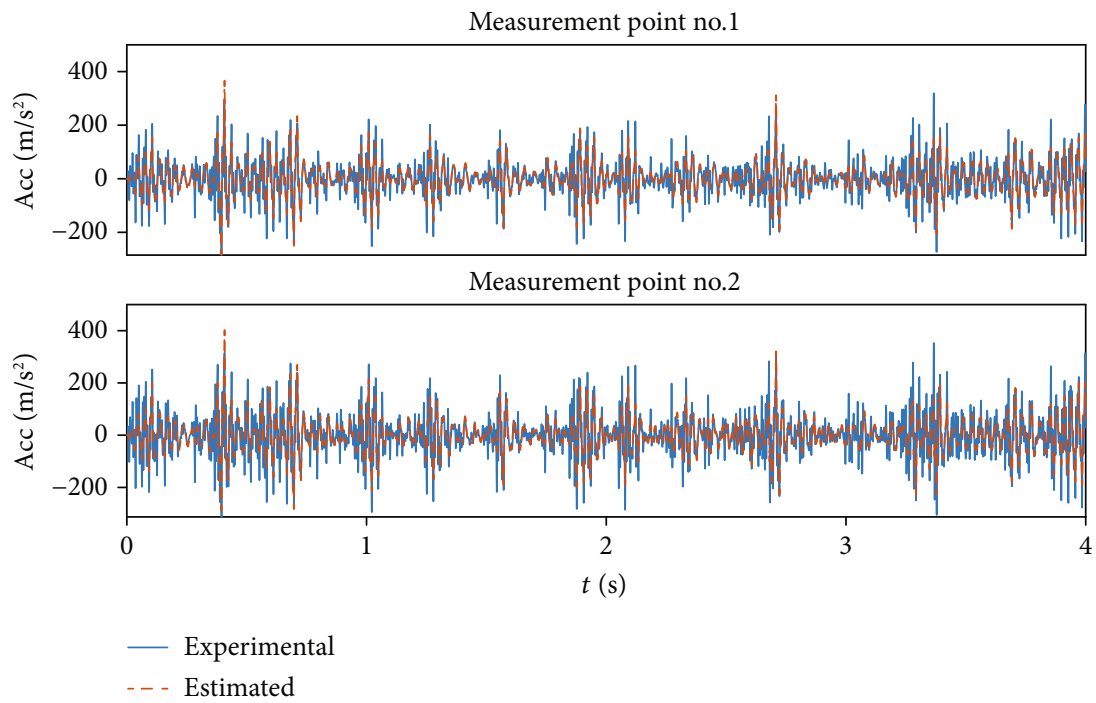

Figure 8: Comparison between the experimental and estimated acceleration signals.

TABLE 1: Natural frequency estimates and mean square errors.

\begin{tabular}{lcc}
\hline Measurement points & Natural frequency estimates (Hz) & Mean square errors \\
\hline No. 1 and no. 2 & $p_{1}=30.531, p_{2}=52.718$ & MSE $_{1}=0.3038, \mathrm{MSE}_{2}=0.3784$ \\
No. 1 and no. 3 & $p_{1}=29.409, p_{2}=84.199$ & $\mathrm{MSE}_{1}=0.2380, \mathrm{MSE}_{3}=0.2326$ \\
No. 1 and no. 4 & $p_{1}=29.180, p_{2}=53.865$ & $\mathrm{MSE}_{1}=0.2716, \mathrm{MSE}_{4}=0.1292$ \\
No. 1 and no. 5 & $p_{1}=30.451, p_{2}=73.201$ & $\mathrm{MSE}_{1}=0.2777, \mathrm{MSE}_{5}=0.4462$ \\
No. 1 and no. 6 & $p_{1}=29.475, p_{2}=55.232$ & $\mathrm{MSE}_{1}=0.2750, \mathrm{MSE}_{6}=0.5444$ \\
No. 2 and no. 3 & $p_{1}=29.405, p_{2}=78.525$ & $\mathrm{MSE}_{2}=0.2790, \mathrm{MSE}_{3}=0.2886$ \\
No. 2 and no. 4 & $p_{1}=28.896, p_{2}=60.396$ & $\mathrm{MSE}_{2}=0.3380, \mathrm{MSE}_{4}=0.0959$ \\
No. 2 and no. 5 & $p_{1}=30.363, p_{2}=73.584$ & $\mathrm{MSE}_{2}=0.3162, \mathrm{MSE}_{5}=0.4480$ \\
No. 2 and no. 6 & $p_{1}=29.310, p_{2}=57.650$ & $\mathrm{MSE}_{2}=0.3648, \mathrm{MSE}_{6}=0.5145$ \\
No. 3 and no. 4 & $p_{1}=32.525, p_{2}=56.198$ & $\mathrm{MSE}_{3}=0.5485, \mathrm{MSE}_{4}=0.2488$ \\
No. 3 and no. 5 & $p_{1}=30.466, p_{2}=72.864$ & $\mathrm{MSE}_{3}=0.3855, \mathrm{MSE}_{5}=0.4404$ \\
No. 3 and no. 6 & $p_{1}=32.977, p_{2}=51.764$ & $\mathrm{MSE}_{3}=0.6263, \mathrm{MSE}_{6}=0.6759$ \\
No. 4 and no. 5 & $p_{1}=34.096, p_{2}=76.609$ & $\mathrm{MSE}_{4}=0.2522, \mathrm{MSE}_{5}=0.4258$ \\
No. 4 and no. 6 & $p_{1}=32.216, p_{2}=57.024$ & $\mathrm{MSE}_{4}=0.1886, \mathrm{MSE}_{6}=0.5556$ \\
No. 5 and no. 6 & $p_{1}=44.720, p_{2}=91.888$ & $\mathrm{MSE}_{5}=0.3265, \mathrm{MSE}_{6}=0.6153$ \\
\hline
\end{tabular}

TABLE 2: Mean square errors with increasing number of measurement points.

\begin{tabular}{lc}
\hline Measurement points & Mean square errors \\
\hline Nos. 1 and 2 & $\mathrm{MSE}_{1}=0.3038, \mathrm{MSE}_{2}=0.3784$ \\
Nos. 1, 2, and 3 & $\mathrm{MSE}_{1}=0.2062, \mathrm{MSE}_{2}=0.2417, \mathrm{MSE}_{3}=0.1926$ \\
Nos. 1, 2, 3, and 4 & $\mathrm{MSE}_{1}=0.1742, \mathrm{MSE}_{2}=0.2041, \mathrm{MSE}_{3}=0.1467, \mathrm{MSE}_{4}=0.0567$ \\
Nos. 1, 2, 3, 4, and 5 & $\mathrm{MSE}_{1}=0.1627, \mathrm{MSE}_{2}=0.1870, \mathrm{MSE}_{3}=0.1342, \mathrm{MSE}_{4}=0.0523, \mathrm{MSE}_{5}=0.1927$ \\
Nos. 1, 2, 3, 4, 5, and 6 & $\mathrm{MSE}_{1}=0.0706, \mathrm{MSE}_{2}=0.0631, \mathrm{MSE}_{3}=0.0565, \mathrm{MSE}_{4}=0.0404 \mathrm{MSE}_{5}=0.0747, \mathrm{MSE}_{6}=0.1240$ \\
\hline
\end{tabular}

modes and to select the best estimates for the physical poles, the idea of a stabilization diagram in traditional modal analysis is used here [23]. Figure 9 depicts the natural frequencies of the identified model with an increasing model order (dots), along with the fast Fourier transform (FFT) spectra of the six experimental acceleration signals (background). 


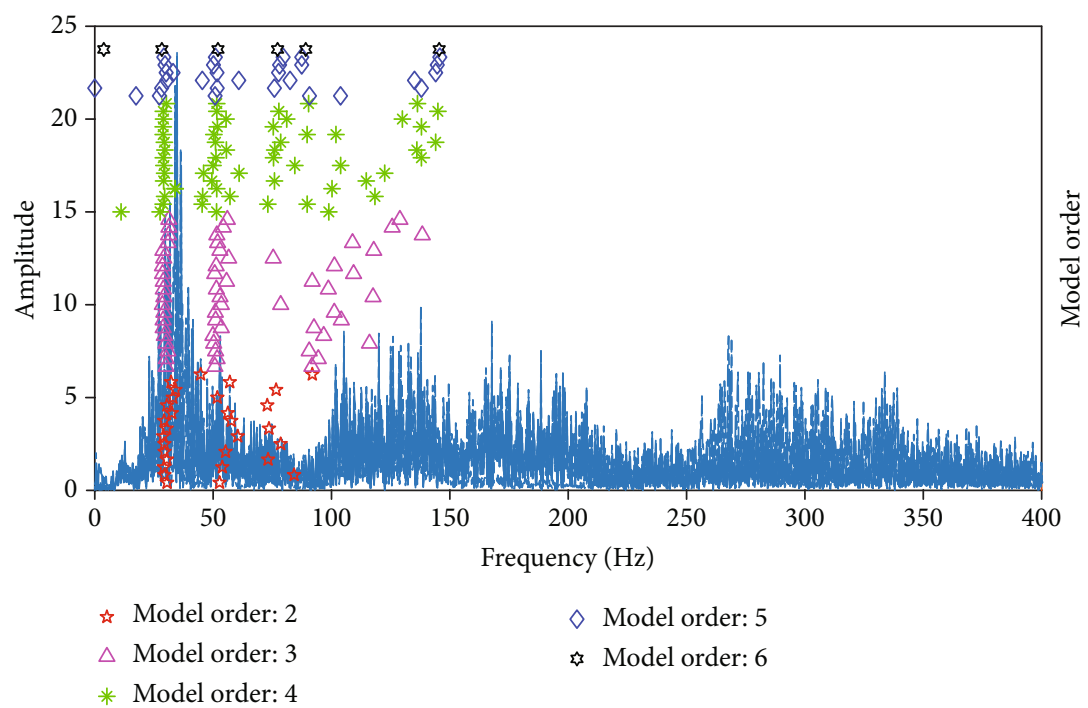

FIGURE 9: Natural frequency estimates and FFT spectra of experimental acceleration signals.

Evidently, the physical poles do not change with an increasing number of assumed poles, and appear as "stable poles" nearly independent of assumed model orders. In contrast to physical poles, computational poles will change with an increasing number of assumed poles in order to model the noise on the measured signals.

As shown in Figure 9, the second natural frequency cannot be well estimated when the identified model order is two. Therefore, data at more measurement points (larger than two) are needed to consider the first two modes of the folding fin. The FFT spectra can be used to indicate the rough values of the natural frequencies, together with the results of the base sine-sweep test. It should be further stressed that natural frequencies of the derived linear system do not agree well with the position where extremum points of the FFT spectra appear, due to the nonlinear internal forces of the true system.

\section{Conclusions}

In this work, the nonlinear dynamic characteristics of a folding fin are experimentally analyzed by using identification approaches. A direct parameter estimation method which can identify the nonlinear system with freeplay under base excitation is proposed and subsequently applied to establish the nonlinear dynamic model of a folding fin. The unknown freeplay nonlinearity of the folding fin is reduced to a polynomial form, and the best set of coefficients is selected by using the significance test, allowing the proposed method to detect and locate the most relevant nonlinearities. Therefore, the method can be used to identify a structure when no a priori knowledge of the type of nonlinearities is given. Furthermore, the proposed method is able to decouple the linear and nonlinear dynamics of a nonlinear system, and estimate natural frequencies of the derived linear system along with nonlinear internal forces in one computational step, which are often required in many aerospace and mechanical structures.

\section{Data Availability}

The data used to support the findings of this study are available from the authors upon request.

\section{Conflicts of Interest}

The authors declare that there are no conflicts of interest.

\section{Acknowledgments}

The authors acknowledge the support from the National Natural Science Foundation of China (Grant Nos. 11802201, 51575378, and 11972245) and the China Postdoctoral Science Foundation (Grant No. 2017M621075).

\section{References}

[1] N. Yang, N. Wang, X. Zhang, and W. Liu, "Nonlinear flutter wind tunnel test and numerical analysis of folding fins with freeplay nonlinearities," Chinese Journal of Aeronautics, vol. 29, no. 1, pp. 144-159, 2016.

[2] D.-K. Kim, J.-S. Bae, I. Lee, and J.-H. Han, "Dynamic model establishment of a deployable missile control fin with nonlinear hinge," Journal of Spacecraft and Rockets, vol. 42, no. 1, pp. 66-77, 2005.

[3] D.-H. Kim and I. Lee, "Transonic and low-supersonic aeroelastic analysis of a two-degree-of-freedom airfoil with a freeplay non-linearity," Journal of Sound and Vibration, vol. 234, no. 5 , pp. 859-880, 2000.

[4] L. Liu and E. H. Dowell, "Harmonic balance approach for an airfoil with a freeplay control surface," AIAA Journal, vol. 43, no. 4, pp. 802-815, 2005.

[5] K. W. Chung, C. L. Chan, and B. H. K. Lee, "Bifurcation analysis of a two-degree-of-freedom aeroelastic system with freeplay structural nonlinearity by a perturbation-incremental method," Journal of Sound and Vibration, vol. 299, no. 3, pp. 520-539, 2007. 
[6] D. Tang and E. H. Dowell, "Aeroelastic airfoil with free play at angle of attack with gust excitation," AIAA Journal, vol. 48, no. 2, pp. 427-442, 2010.

[7] W. Zhang, L.-L. Chen, X.-Y. Guo, and L. Sun, "Nonlinear dynamical behaviors of deploying wings in subsonic air flow," Journal of Fluids and Structures, vol. 74, pp. 340-355, 2017.

[8] X. Guo, Y. Zhang, W. Zhang, L. Sun, and S. Chen, "Nonlinear dynamics of Z-Shaped folding wings with 1:1 inner resonance," International Journal of Bifurcation and Chaos, vol. 27, no. 8, article 1750124, 2017.

[9] J. Xiang, Y. Yan, and D. Li, "Recent advance in nonlinear aeroelastic analysis and control of the aircraft," Chinese Journal of Aeronautics, vol. 27, no. 1, pp. 12-22, 2014.

[10] A. Abdelkefi, R. Vasconcellos, F. D. Marques, and M. R. Hajj, "Modeling and identification of freeplay nonlinearity," Journal of Sound and Vibration, vol. 331, no. 8, pp. 1898-1907, 2012.

[11] J.-S. Bae, D.-K. Kim, W.-H. Shin, I. Lee, and S.-H. Kim, "Nonlinear aeroelastic analysis of a deployable missile control fin," Journal of Spacecraft and Rockets, vol. 41, no. 2, pp. 264-271, 2004.

[12] Z. Wu, N. Yang, and C. Yang, "Identification of nonlinear multi-degree-of freedom structures based on Hilbert transformation," Science China Physics, Mechanics \& Astronomy, vol. 57, no. 9, pp. 1725-1736, 2014.

[13] Z. Wu, N. Yang, and C. Yang, "Identification of nonlinear structures by the conditioned reverse path method," Journal of Aircraft, vol. 52, no. 2, pp. 373-386, 2015.

[14] G. Kerschen, K. Worden, A. F. Vakakis, and J.-C. Golinval, "Past, present and future of nonlinear system identification in structural dynamics," Mechanical Systems and Signal Processing, vol. 20, no. 3, pp. 505-592, 2006.

[15] J. P. Noël and G. Kerschen, "Nonlinear system identification in structural dynamics: 10 more years of progress," Mechanical Systems and Signal Processing, vol. 83, pp. 2-35, 2017.

[16] S. F. Masri and T. K. Caughey, "A nonparametric identification technique for nonlinear dynamic problems," Journal of Applied Mechanics, vol. 46, no. 2, pp. 433-447, 1979.

[17] K. Worden and G. R. Tomlinson, Nonlinearity in Structural Dynamics: Detection, Identification and Modelling, Institute of Physics Publishing, Bristol, 2000.

[18] S. A. Billings and I. J. Leontaritis, "Identification of Nonlinear Systems Using Parametric Estimation Techniques," in The IEE conference on control and its application, pp. 183-187, Warwick, UK, 1981.

[19] S. A. Billings, Nonlinear System Identification: NARMAX Methods in the Time, Frequency, and Spatio-Temporal Domains, John Wiley \& Sons, Chichester, 2013.

[20] S. L. Lacy and D. S. Bernstein, "Subspace identification for non-linear systems with measured-input non-linearities," International Journal of Control, vol. 78, no. 12, pp. 906-926, 2005.

[21] S. Marchesiello and L. Garibaldi, "A time domain approach for identifying nonlinear vibrating structures by subspace methods," Mechanical Systems and Signal Processing, vol. 22, no. 1, pp. 81-101, 2008.

[22] K. S. Mohammad, K. Worden, and G. R. Tomlinson, "Direct parameter estimation for linear and non-linear structures," Journal of Sound and Vibration, vol. 152, no. 3, pp. 471-499, 1992.

[23] W. Heylen, S. Lammens, and P. Sas, Modal Analysis Theory and Testing, Katholieke Universiteit Leuven, Leuven, 2007. 


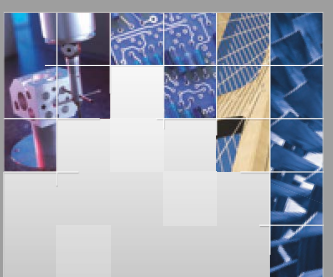

\section{Enfincering}
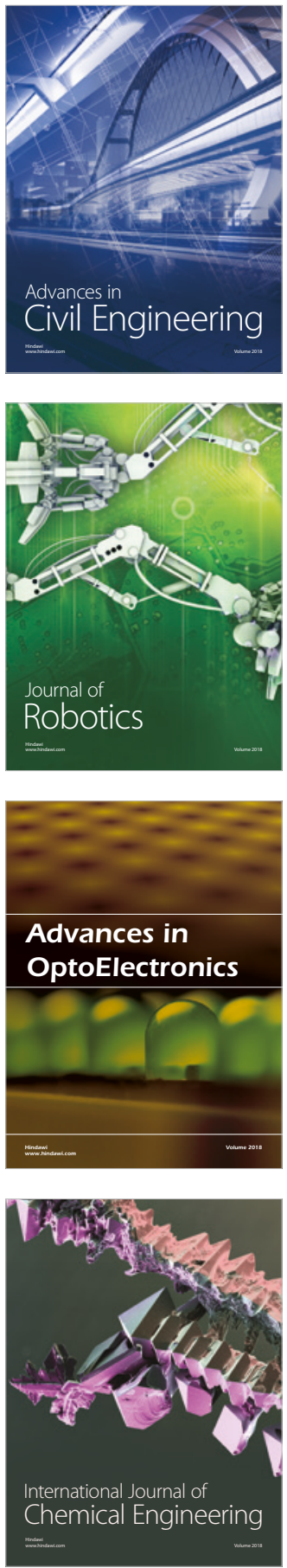

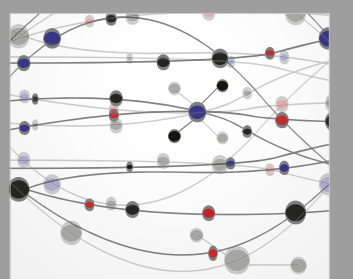

\section{Rotating \\ Machinery}

The Scientific World Journal

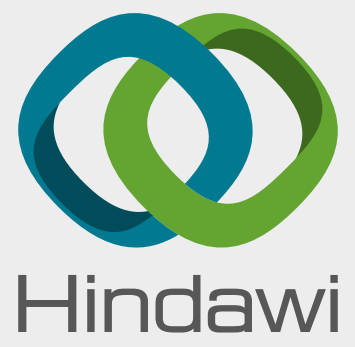

Submit your manuscripts at

www.hindawi.com
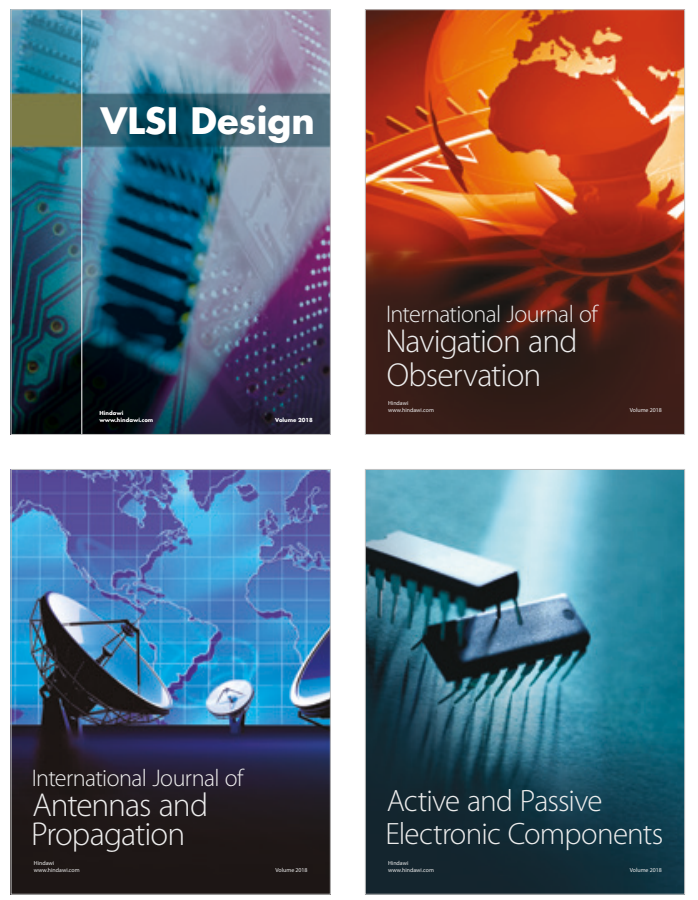
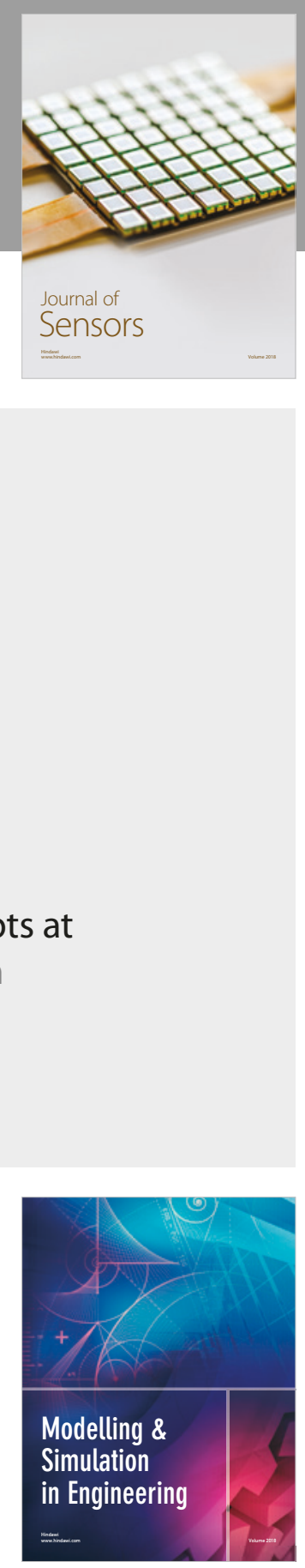

\section{Advances \\ Multimedia}
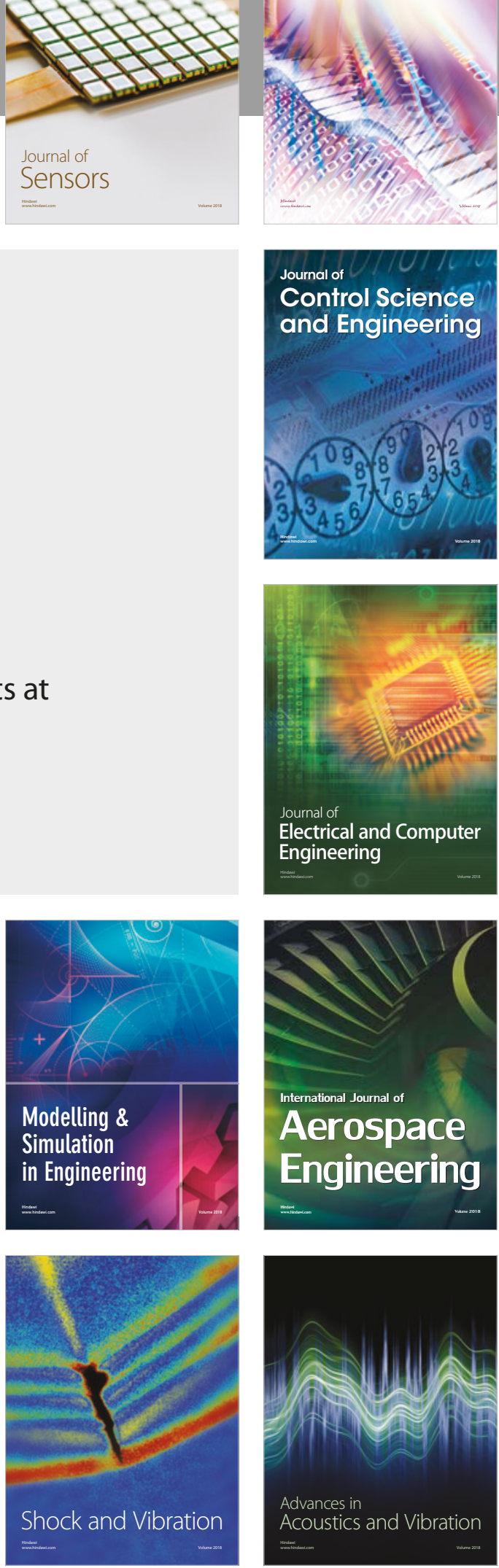